\title{
«Der Patient steht im Zentrum, und dort steht er im Weg!»
}

Interview: Anna Sax

Korrespondenz:

Therese Stutz Steiger

Melchenbühlweg 2

CH-3006 Bern

geschaeftsstelle[at]svoi-asoi.ch

\begin{abstract}
Am 8. Juni findet auf dem Twannberg das dritte Osteogenesis Imperfecta Symposium statt. Die Veranstaltung bietet eine Plattform für den Erfahrungsaustausch zwischen Betroffenen, Medizinerinnen, Physiotherapeuten und weiteren Gesundheits-Fachpersonen. Weil die Osteogenesis Imperfecta - im Volksmund Glasknochen genannt - so selten vorkomme, seien interdisziplinärer Austausch und Selbsthilfe umso wichtiger, betont Therese Stutz Steiger, Co-Präsidentin der SVOI, im Interview mit der Schweizerischen Ärztezeitung.
\end{abstract}

\section{Wer ist die SVOI und was sind ihre Ziele?}

Therese Stutz Steiger: Die Schweizerische Vereinigung Osteogenesis Imperfecta (SVOI) wurde 1986 als gesamtschweizerischer Selbsthilfeverein für Menschen mit Glasknochen und deren Angehörige gegründet. Sie hat rund 150 Mitglieder, hauptsächlich Einzelpersonen und Familien. Die SVOI ist selbst Mitglied bei einigen Behinderten- und Patientenorganisationen, zum Beispiel bei ProRaris, Agile und der Rheumaliga Schweiz. Unser Ziel ist die optimale

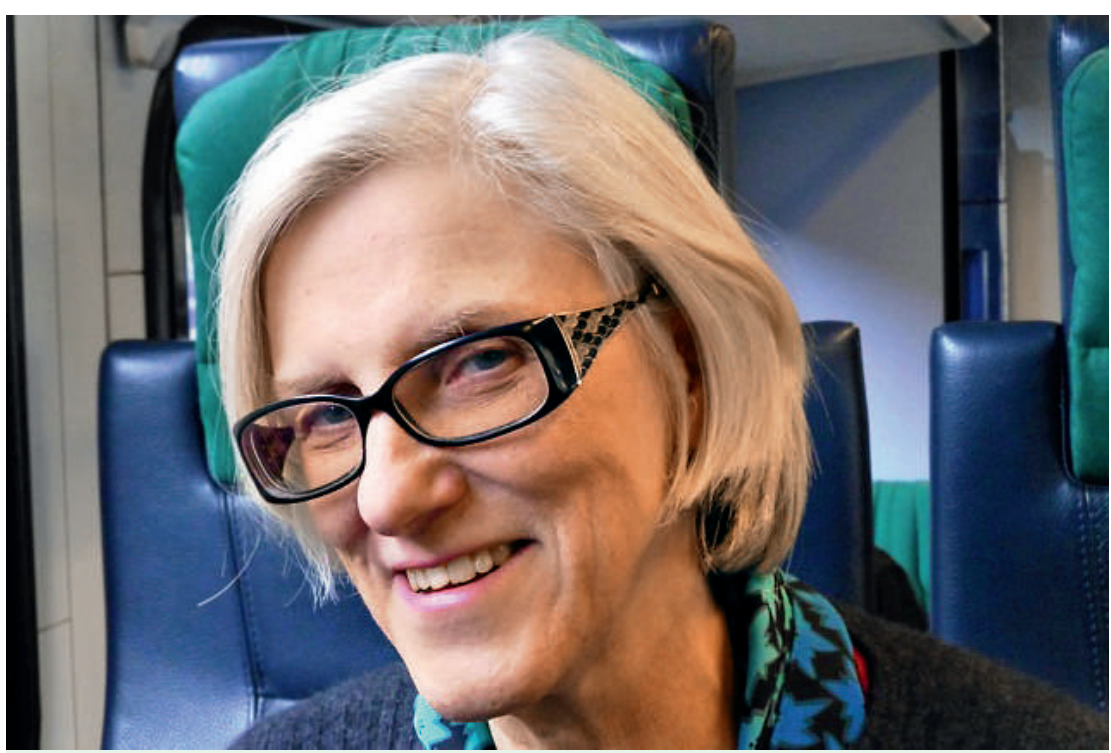

Therese Stutz Steiger ist Ärztin und Public Health Consultant. Nach dem Doktorat absolvierte sie ein Nachdiplomstudium an der London School of Hygiene and Tropical Medicine mit Master-Abschluss in Community Medicine. Von 1986 bis 2010 war sie in verschiedenen Funktionen im Bundesamt für Gesundheit tätig. 2012 wurde sie zum Ehrenmitglied von Public Health Schweiz gewählt. Therese Stutz Steiger ist selbst OI-Patientin und Autorin zahlreicher Publikationen, u.a. zum Thema Gesundheitskompetenz.
Integration von Menschen mit OI in Gesellschaft, Schule und Arbeitswelt. Wir wollen die bestmögliche medizinische Beratung und Versorgung vermitteln. Wichtig ist uns auch eine aktive Betreuung, Unterstützung und Vernetzung unserer Mitglieder.

Die SVOI will Mediziner, Paramedizinerinnen und OIBetroffene miteinander vernetzen: Weshalb ist das wichtig?

OI ist eine seltene Krankheit, 300-400 Betroffene gibt es in der Schweiz. Die wenigsten Ärzte sind Spezialisten auf diesem Gebiet. Physiotherapeutinnen und Patienten wissen oft mindestens so viel über OI. Deshalb braucht es den interdisziplinären Austausch.

Mit dem OI-Symposium vom 8. Juni wollen Sie diesen Austausch fördern. Wie sehen Sie dabei die jeweilige Rolle der Medizinerinnen, der Paramediziner und der Patientinnen?

Die Ärzte kommen zum Teil als Referenten und Auskunftspersonen, zu einem anderen Teil als Lernende und zum Erfahrungsaustausch. Wir hoffen, dass Hausärztinnen von Betroffenen teilnehmen, denn Hausärzte und Spezialistinnen kommen viel zu selten miteinander ins Gespräch. Gesundheitsfachleute werden von ihren Erfahrungen mit OI-Patientinnen berichten, aber sie werden sicher auch etwas lernen können. Unter anderem wird es eine Demonstration einer Physiotherapeutin mit einem Patienten zur Halliwick Wassertherapie geben. Die Patientinnen schliesslich werden Gelegenheit erhalten, Neues über ihre Krankheit zu lernen, Fragen zu stellen und eigene Erfahrungen beizusteuern.

Sie führen dieses Symposium schon zum dritten Mal durch: Was haben die beiden vorhergehenden Veranstaltungen gebracht?

Am ersten und zweiten Symposium kamen neben medizinischen auch nichtmedizinische Themen zur Sprache, zum Beispiel alternative Schmerztherapien, Versicherungen, Schulkarriere, Jobsuche, Reisen, Sport, Familienplanung und Wechseljahre. Die beiden Veranstaltungen boten zugleich Gelegenheit zur «Fachleute-Edukation». 


\section{Was genau meinen Sie damit?}

In unserer Vereinigung ist die Kooperation zwischen Laien und Fachpersonen sehr weit fortgeschritten. Dazu haben die bisherigen Symposien viel beigetragen. OI ist selten, so dass die Patienten selber dafür sorgen müssen, dass zwei Ärztinnen miteinander sprechen oder im Spital eine interdisziplinäre Sprechstunde stattfindet. Wir sind unsere eigenen CaseManager. Das ist manchmal harte Arbeit. Dank der SVOI und dem geplanten Beirat, von dem eine Vorform schon seit Jahren existiert, kommen Fachpersonen und Patientinnen einmal im Jahr zusammen und sprechen miteinander. Das ist nicht selbstverständlich, und wir sind auch stolz darauf, dass wir so gutvernetzt sind und für uns selbst einstehen können.

\section{«Wir sind unsere eigenen}

\section{Case-Manager.»}

Sie sind zugleich Ärztin und Patientin. Sind Sie damit für eine Rolle als Vermittlerin prädestiniert?

Ich bin von OI betroffen, was zur Folge hatte, dass ich vor allem als Kind sehr häufig Patientin war; die «Innensicht» ist mir also vertraut. Als Ärztin kann ich aber auch den Standpunkt der «Aussensicht» nachvollziehen, und meine Spezialausbildung in Public Health hat dazu geführt, dass ich weniger den Einzelmenschen in seiner Rolle als Patient als die Bevölkerung als Ganzes in den Blick nehme. Besonders interessiert mich dabei das Verhältnis zwischen Selbsthilfe und professioneller Betreuung. Meine Rolle als Vermittlerin sehe ich hauptsächlich darin, den Dialog zwischen Fachleuten und Betroffenen zu fördern.

\section{Was braucht es, damit dieser Dialog sich verbessert?}

Bei den Ärztinnen braucht es primär den Willen, gut zu kommunizieren und falls nötig auch entsprechende Fortbildung zu betreiben. Sie müssen fähig sein, sich vom Fachjargon zu lösen und trotzdem die Patientinnen für voll zu nehmen. Die Patienten sind Experten mit Erfahrungswissen, das eine wichtige Ergänzung zum Fachwissen sein kann. Sie brauchen eine gesunde Neugier und die Fähigkeit, Informationen zu recherchieren und zu interpretieren, dabei wenn möglich «cool» zu bleiben und beim Kontakt mit der Fachperson beharrlich Fragen zu stellen. Beide miteinander müssen Abmachungen treffen, die es ermöglichen, für den Patienten realisierbare Therapieschritte einzuhalten.
Finden die Interessen der Patientinnen und Patienten in der Gesundheitspolitik die Beachtung, die sie verdienen?

Nein, leider nicht. «Der Patient steht im Zentrum, und dort steht er im Weg», sagte einst ein Kollege von mir im BAG, und er hat leider noch immer recht. Im Finanzgerangel zwischen Krankenkassen, Leistungserbringern, Kantonen und Spitälern geht die Patientin mit ihren primären Interessen meistens vergessen.

Welches sind die besonderen Probleme und Bedürfnisse von Menschen mit seltenen Krankheiten?

Der Austausch mit ihresgleichen und die Suche nach Ärzten und Therapeutinnen mit dem nötigen Spezialwissen ist erschwert. Wenn teure Therapien notwendig sind, kann es zu massiven Problemen mit der Krankenkasse kommen. Patienten stossen auf Unverständnis oder können gar psychiatrisiert werden. ProRaris, die Allianz seltener Krankheiten, macht seit ihrem Start 2010 auf diese Probleme aufmerksam. Sie vertritt in der Schweiz bereits 45 Organisationen mit insgesamt fast 20000 Mitgliedern und ist damit in nur drei Jahren zur grössten Patientenorganisation geworden.

Was möchten Sie den Leserinnen und Lesern der Ärztezeitung besonders ans Herz legen?

Ärzte neigen dazu, im Namen der Patientinnen zu sprechen. Vor der Managed-Care-Abstimmung sagte die FMH: «Unsere Patienten sind dagegen», und die Hausärzte sagten: «Unsere Patienten sind dafür.» Die Ärzte sollten lernen, die Patientinnen für sich selber sprechen zu lassen. Sie sollten sich vermehrt mit Patientenorganisationen auseinandersetzen und ihre Patienten auf sie aufmerksam machen. Das Bewusstsein für die Aufgabe, Kompetenzen zu fördern, sollte in allen therapeutischen Beziehungen vorhanden sein.

\section{OI-Symposium vom 8. Juni 2013}

Die SVOI/ASOI lädt zum 3. OI-Symposium Betroffene, Fachpersonen und Interessierte zu einer interdisziplinären Tagung auf den Twannberg (BE) ein. Highlights sind Berichte über erste Erfahrungen mit Rehabilitationsprogrammen für Kinder und Erwachsene, erste Langzeiterfahrungen mit Bisphosphonattherapien, Frakturheilungen und orthopädische Interventionen («Heilt's oder heilt's nicht?») und Wirbelsäulenkorrekturen («Hält's oder hält's nicht?»). Programm und Anmeldung: www.svoi-asoi.ch. Die Orthopädische Fachgesellschaft hat acht Creditpunkte zugeteilt. 